\title{
Catégorisation, classifiance et organisation des séquences textuelles
}

\author{
Guérin, Olivia \\ EA 2290 SYLED / Fédération Clesthia \\ Université Paris 3 \\ olivia.guerin@univ-paris.fr
}

Les études des vingt dernières années consacrées à la construction textuelle de la référence (par exemple Charolles \& Schnedecker 1993, Kleiber et al. éd. 1997, Schnedecker 1998, Kleiber 2001 b, De Mulder \& Schnedecker éd. 2001) ont permis de décrire finement le fonctionnement des chaînes de référence et leur agencement au sein des textes. Nous souhaitons aborder ce type de questionnements en éclairant l'étude de la séquentialité par celle des contraintes éventuelles qui peuvent peser sur l'usage des formes nominales. La présente réflexion portera donc sur les procédures de nomination et de catégorisation telles qu'elles sont mises en œuvre dans la séquentialité textuelle.

Notre hypothèse est que toutes les formes nominales ne jouent pas le même rôle dans la construction textuelle de la référence, et que ceci tient à leurs propriétés sémantico-référentielles : elles n'instaurent pas les mêmes types de catégorisation. Nous tenterons de montrer que certaines des contraintes d'emploi des formes nominales au sein d'une séquence textuelle peuvent précisément s'expliquer par ces fonctionnements sémantico-référentiels différenciés. Dans ce parcours, nous aurons à questionner certains modèles récents de la catégorisation en discours, en particulier les approches constructivistes de la référence et l'étude ethnométhodologique des catégorisations. Certes, notre approche présente une affinité d'objet avec ces perspectives, puisqu'il s'agit pour nous d'étudier la catégorisation non pas d'un strict point de vue lexical ${ }^{1}$, mais telle qu'elle se déploie au plan textuel et discursif; cependant, nous proposerons une conception de la catégorisation et de son rôle dans la construction de la référence assez différente de celle développée au sein de ces approches. Pour nous, il est essentiel, même lorsque l'on travaille sur les discours, de rendre compte des contraintes linguistiques d'emploi des formes permettant d'effectuer la catégorisation. Dès lors, nous proposerons une approche plus strictement séquentielle des faits de catégorisation. Pour illustrer notre propos, nous nous appuierons sur l'analyse de séquences descriptives, qui font apparaître ces différences de valeurs et ces contraintes d'emploi.

\section{De l'instabilité des catégorisations à la construction de l'intercompréhension}

La ligne de partage entre sémanticiens objectivistes et subjectivistes s'est vue réactivée dans la dernière décennie du $20^{\mathrm{e}}$ siècle par des débats opposant conceptions constructivistes de la référence et approches plus traditionnellement référentialistes - notamment la théorie des référents évolutifs ${ }^{2}$. Ces deux approches développent des conceptions antithétiques des procédures référentielles.

Par opposition à la conception, centrale en sémantique référentielle, selon laquelle les expressions linguistiques renverraient à des entités du monde réel indépendantes du langage, se sont développés divers modèles sémantiques aréférentiels, comme la sémantique indexicale de Cadiot (notamment Cadiot \& Habert 1997, Cadiot \& Nemo 1997, Cadiot \& Lebas 2003), ou encore les approches constructivistes de la référence (notamment Apothéloz \& Reichler-Béguelin 1995, Berthoud 1999) ${ }^{3}$. Ces conceptions reprochent à la sémantique référentielle son objectivisme, et développent au contraire l'idée selon laquelle le sens n'a rien de préétabli, qu'il est le résultat d'une perpétuelle renégociation discursive (Kleiber 1997).

Pour le paradigme constructiviste, qui a trouvé diverses applications dans le champ des études sémantiques ces dernières décennies, la réalité n'a aucune existence objective ${ }^{4}$. Le monde est toujours un 
monde perçu, façonné par notre perception, notre culture et nos interactions. Les constructivistes reprochent tout particulièrement au paradigme objectiviste la conception selon laquelle il y aurait d'un côté le monde, et de l'autre côté le langage qui le nomme ; ceci revient à concevoir les langues comme des nomenclatures, là où l'approche constructiviste considère que le monde ne préexisterait pas au discours. Ainsi, un même objet peut être catégorisé de diverses manières, en fonction de la situation discursive ; Dubois \& Mondada (1995) empruntent à Barsalou (1983) l'exemple du PIANO, qui peut être catégorisé de diverses manières selon les situations pratiques : comme instrument de musique, comme meuble lourd pour un déménageur, ou encore comme chose à emporter de la maison en feu lors d'un incendie. Le langage ne peut être considéré comme un simple répertoire d'étiquettes posées sur des réalités ayant une existence autonome. Ainsi, la catégorisation ne peut pas se fonder sur l'« ontologie »du référent; elle ne s'opère pas par validation des critères d'applicabilité référentielle enregistrés dans une dénomination, contrairement à ce qu'avance la sémantique lexicale ${ }^{5}$. La catégorisation est conçue dans cette perspective comme une propriété liée aux termes lexicaux, mais comme une activité discursive dynamique.

Déniant l'idée qu'il existe des référents, l'optique constructiviste substitue à cette dernière notion, jugée fixiste et essentialisante, celle d'objet de discours. Elle considère que le discours ne renvoie pas à des objets prédécoupés : il construit de pures représentations mentales, qui n'ont d'existence que discursive, et n'ont aucun statut extralinguistique. L'objet de discours, qui constitue, dans la définition donnée par Apothéloz \& Reichler-Béguelin (1995), un ensemble évolutif d'informations incluses dans le savoir partagé des interlocuteurs ${ }^{6}$. La référenciation ${ }^{7}$ est conçue comme foncièrement instable; dans cette opération, un objet de discours est construit par des catégorisations multiples au fil des activités verbales, aucune des formes permettant de catégoriser un même objet de discours n'ayant plus de validité qu'une autre. L'objet de discours est précisément construit par le parcours des diverses formes permettant de le catégoriser $^{8}$, comme cherchent à le montrer Apothéloz \& Reichler-Béguelin (1995) à partir de cet exemple emprunté à Ponge pour illustrer leur conception aréférentielle de la catégorisation :

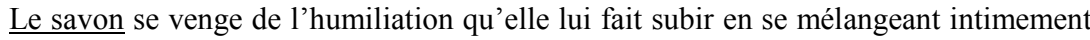
à l'eau, en s'y mariant de la façon la plus ostensible. Cet oeuf, cette plate limande, cette petite amande se développe rapidement en poisson chinois. (F. Ponge, «Le savon ", Le parti pris des choses)

Pour les auteurs, l'objet de discours est ici construit tout autant par les formes de reprise cet ouf, cette plate limande, cette petite amande que par la forme initiale le savon. Ainsi, toutes les catégorisations se vaudraient et joueraient un rôle équivalent dans la construction de l'objet de discours.

À partir de ce type d'analyses, ces approches développent l'idée que les formes nominales n'ont pas d'applicabilité référentielle fixe. Cette conception semble remettre en question l'idée qu'il puisse exister un sens lexical partagé pour les dénominations, sens qui, pour la sémantique référentielle, est au fondement de la catégorisation. Certes, on peut reconnaître que le modèle de la catégorisation proposé par la sémantique référentielle et lexicale est trop restrictif en ce qu'il ne rend pas compte de la catégorisation telle qu'elle fonctionne dans les discours. De fait, son objectif est de rendre compte de la délimitation des catégories enregistrées dans les dénominations lexicales, des conditions d'applicabilité référentielle de ces dernières et de l'organisation hiérarchique du lexique. Ainsi, elle ne rend compte que des emplois standard des dénominations, là où les approches discursives, centrées sur la manière dont les discours construisent des représentations, s'intéressent à des emplois moins typiques des formes nominales (comme ouf pour renvoyer au savon dans l'exemple ci-dessus) et à la manière dont elles concourent à l'élaboration de ces représentations.

Une optique similaire à celle développée dans les études constructivistes nous semble se retrouver dans l'approche ethnométhodologique ${ }^{9}$ de la catégorisation appliquée aux problèmes de référence, qui présente des recoupements avec cette perspective sur la conception de la référenciation et de l'objet de discours ${ }^{10}$. On insiste dans cette perspective sur le fait qu'au sein d'un extrait conversationnel (ou d'une séquence textuelle quand la méthode est appliquée à des textes), un même objet de discours est construit par le parcours des diverses formes qui y renvoient. 
Étudiant les procédures de verbalisation de l'espace dans les récits de voyage, Mondada (1994) met l'accent sur l'instabilité foncière des catégorisations. Le récit de voyage, comparé par l'auteure aux notes de terrain des anthropologues, est conçu comme un discours où le savoir n'est pas encore formulé définitivement, mais où il est en cours d'élaboration. Ainsi les objets de discours s'y caractériseraient par leur déformabilité, marquée par des catégorisations multiples au sein d'une même description :

Fidèle à ma coutume, je me suis dirigée vers le port. Le port !... hélas ! c'est un mur haut de trente pieds, sur lequel des soldats ou des bateliers affrontent seuls les vertiges ; il fallait, pour grimper là, une fermeté que je n'ai point, et, retournant sur mes pas, j'ai quitté ces faux semblans de quais, pour me renfermer avec mes souvenirs dans l'appartement... c'est-à-dire la salle voûtée, obscure, large et longue, à ne pas y reconnaître son père, qu'on m'a donnée en guise de chambre à coucher. (A. E. de Gasparin Voyage d'une ignorante dans le midi de la France et l'Italie, 1835, cité in Mondada 1994)

À partir de ce type de faits, on a pu tirer la conclusion selon laquelle aucune forme de catégorisation n'est plus valable qu'une autre pour catégoriser un objet de discours (idée développée tant chez Apothéloz \& Reichler-Béguelin que chez Dubois \& Mondada 1995). Dès lors, on avance que les catégorisations sont foncièrement $a d h o c$, instables, voire sans cesse renégociables en discours.

Si l'on peut admettre sans difficulté l'idée que les diverses formes nominales convoquées dans ce même extrait concourent à la construction d'une représentation complexe, il convient selon nous d'interroger l'affirmation selon laquelle aucune des diverses catégorisations opérées pour construire un même objet de discours n'aurait plus de validité qu'une autre, que les catégorisations sont sans cesse instables et renégociables. Au contraire, nous tenterons dans la suite de cette étude de montrer que divers niveaux de contraintes influent sur l'emploi des formes permettant la catégorisation, et que toutes ne sont pas aptes à construire la référence de la même manière.

De fait, les thèses constructivistes ont fait l'objet de diverses critiques. Des auteurs comme Lärsson (1997, 2008) et Kleiber (1997) ont montré qu'elles étaient trop radicales. Certes, des zones d'instabilité apparaissent dans les phénomènes référentiels; mais il existe du sens stable dès lors qu'il y a intersubjectivité, dès lors que le sens est partagé au sein d'une communauté linguistique. Ainsi, le sens n'est jamais entièrement fondé ni sur la subjectivité, ni sur l'objectivité radicales. Kleiber (1997) insiste en outre sur la nécessité d'un retour à une sémantique référentielle : le sens n'est pas infiniment variable, notamment parce qu'il est «branché » sur la référence ; quand on parle, on parle bien de quelque chose, qui a une existence en dehors du langage, et non pas de pures représentations mentales. Aussi est-il nécessaire de tenir compte du référent dans l'analyse du sens.

La dimension de l'intersubjectivité nous semble essentielle à prendre en compte, tout particulièrement lorsque l'on travaille sur des textes écrits ${ }^{11}$. La construction textuelle de la référence nous semble impliquer un nécessaire appui sur des représentations partagées. Si les catégorisations étaient foncièrement instables, si toutes les formes nominales pouvaient être indifféremment employées pour renvoyer à l'objet de discours construit dans un texte écrit - et plus particulièrement dans les séquences descriptives qui ont pour objectif de faire visualiser un objet ${ }^{12}-$, le lecteur, qui n'a pas d'accès direct à la situation, pourrait-il se le représenter? Selon nous, les approches mettant l'accent sur la fluctuance des catégorisations prennent insuffisamment en compte la nécessaire construction de l'intercompréhension. Nous pensons que dans le texte écrit, et tout particulièrement dans les séquences descriptives, il existe des contraintes d'emploi des formes qui permettent de garantir cette intercompréhension. La référence se fonde partiellement dans les textes sur du partagé, certaines formes jouant un rôle de fixation de la référence. Les approches mettant l'accent sur la fluctuance du sens prennent selon nous insuffisamment en compte le paramètre de la nécessaire construction de l'intercompréhension.

Ainsi, nous tenterons pour notre part de rendre compte du rôle des formes de catégorisation dans la construction textuelle de la référence en accordant du crédit à l'extralinguistique et au caractère partagé du sens. Notre démarche se distanciera tant d'une approche strictement lexicale et référentielle de la catégorisation - nous ne nous intéressons non pas seulement, en effet, à la catégorisation telle qu'elle est effectuée par le lexique, mais telle qu'elle se déploie en discours et dans la textualité, - que des modèles 
constructivistes et aréférentiels dont nous venons de rappeler les principaux présupposés. L'idée, développée au sein de ces derniers modèles, d'une instabilité de la catégorisation et de la construction $a d$ hoc des objets de discours nous semble en effet s'appuyer sur une conception spécifique de l'opération de catégorisation qui mérite d'être interrogée.

Ces approches passent selon nous sous silence un certain nombre de contraintes qui pèsent sur la construction de la référence au sein des textes et des discours. Nous pensons que pour analyser ce type de catégorisation, il faut tenir compte de quatre types de paramètres qui influent sur les procédures de catégorisation : (1) la construction de l'intercompréhension ; (2) l'influence du référent sur les pratiques de catégorisation; (3) les propriétés de langue des formes permettant la catégorisation ; (4) les contraintes textuelles sur l'emploi de ces formes.

\section{Réévaluation de la notion de classifiance en termes d'emplois discursifs}

Afin de porter au jour cette imbrication de contraintes relevant de différents niveaux (référentiel, linguistique, textuel et discursif), nous allons tout d'abord nous intéresser aux fonctionnements sémantico-référentiels effectifs des expressions servant à catégoriser dans les textes et les discours. Nous pensons en effet que toutes les formes nominales n'opèrent pas les mêmes types de catégorisation, du fait de propriétés sémantico-référentielles différenciées, ce qui a pour conséquence qu'elles n'ont pas les mêmes lattitudes d'emploi au sein des séquences textuelles.

Pour mettre en lumière les fonctionnements différenciés qu'assume la catégorisation au plan textuel, nous partirons de la notion de classifiance, en la réorientant dans une optique discursive. Ceci nous permettra d'analyser l'incidence des formes classifiantes au sein des séquences descriptives, et de mettre en lumière leur rôle de stabilisation de la référence.

Bien que localement, les catégorisations mises en œuvre en discours puissent parfois sembler instables et négociables, dès lors que l'on s'intéresse aux modes de construction de la référence au plan de la séquence textuelle, on voit au contraire apparaître des phénomènes qui tendent vers la stabilisation de la référence.

Nous tenterons de mettre en lumière l'hétérogénéité des phénomènes dont il est rendu compte au moyen du terme de catégorisation en reformulant dans une optique discursive la distinction proposée par Milner (1978) entre formes classifiantes et formes non classifiantes.

Dans la perspective de Milner, un nom a soit le statut de forme classifiante (un nom comme professeur indique l'appartenance du référent à une classe), soit de forme qualifiante (les noms « de qualité » ne classifient pas : Tu es un imbécile). Ces statuts sont dans cette optique conçus comme intrinsèques aux formes : une forme est par nature soit classifiante, soit qualifiante. Si cette distinction est valide, il nous semble utile de la réaménager pour rendre compte du fonctionnement en discours des formes nominales. Notons que certaines formes nominales sont certes par nature classifiantes : une dénomination telle que linguiste, dans ses emplois prototypiques, classifie (Je suis linguiste). Cependant, dans certains emplois, la forme peut assumer également une valeur qualifiante. Il suffit qu'un tel terme soit employé dans une structure syntaxique typique de l'insulte pour qu'il devienne qualifiant : dans Espèce de linguiste ! le nom linguiste ne sert plus seulement à indexer un métier, mais également à qualifier (à déprécier) la personne visée. Des emplois dérivés, hors du strict champ de la classification, sont donc possibles.

Hormis le cas des insultes, d'autres types d'emplois mettent l'accent sur la valeur de qualification du nom : c'est le cas dans des énoncés du type Il fait vraiment très prof, ou Il a encore fait son prof toute la soirée, où il ne s'agit pas uniquement d'indiquer l'appartenance à une classe, mais bien une qualité (en l'occurrence, un type d'attitude, de comportement). On note que ce sont les structures syntaxiques d'emploi (dans les exemples donnés, soit les enclosures du type espèce de, soit les structures verbales faire très $x$, faire son $x$ ) qui activent la valeur qualifiante et font passer la valeur classifiante au second 
plan $^{13}$, ce qui tient au fait que dans ces emplois, le nom apparaît non plus en emploi référentiel, mais prédicatif.

Ces observations amènent à penser que cette distinction entre formes classifiantes et formes qualifiantes doit être revue non pas en termes de statuts intrinsèques des formes, mais en terme de fonctionnements en discours ; on pourrait parler d'emploi classifiant ou d'emploi qualifiant d'un nom.

Nous faisons l'hypothèse que la sémantique référentielle d'une part, les approches constructiviste et ethnométhodologique d'autre part ne parlent pas des mêmes objets sous le même terme de catégorisation. Cette distinction entre emplois classifiants et emplois qualifiants permet de confirmer cette hypothèse.

La catégorisation telle qu'elle est conçue en sémantique référentielle, lexicale et cognitive est indissociable de la notion de classifiance. Les exemples privilégiés par les études sémantiques de ce type pour décrire les mécanismes de catégorisation sont des formes telles que oiseau ou chien, qui sont généralement conçues comme des catégories lexicales ou des catégories sémantiques (par exemple Nyckees 1998, ou Dubois \& Poitou 1999). Les auteurs se situant dans cette optique s'intéressent à des concepts en tant qu'ils sont portés par des mots du lexique : il s'agit de fait de s'interroger sur les conditions d'applicabilité référentielle des dénominations correspondantes. On se demande par exemple si une autruche peut être appelée oiseau, dans la mesure où elle semble se situer à la marge de la catégorie oiseau, dont elle constitue un exemplaire atypique. On analyse des formes nominales en tant qu'elles permettent la classification des éléments de l'expérience. Il est en outre à noter que ces études s'intéressent uniquement aux emplois standard des dénominations. En effet, elles ne traitent pas de leurs emplois dérivés : les analyses ne portent pas sur des emplois métaphoriques du type Paul est vraiment un chien, C'est un drôle d'oiseau, ou Arrête de faire l'autruche, où ces noms, d'espèces à l'origine, n'apparaissent pas en emploi classifiant, mais qualifiant. Ainsi, la catégorisation est conçue dans cette perspective comme un problème de classement des entités dans la grille des savoirs sur le monde et de lexicalisation de ces savoirs. Les formes analysées sont systématiquement des formes classifiantes, en emploi standard.

La délimitation même du phénomène de catégorisation est bien différente dans les approches constructiviste et ethnométhodologique. On considère dans cette perspective que le locuteur dispose d'une série non close de formes pour catégoriser un objet de discours. Ces formes peuvent être des dénominations lexicales en emploi standard (chien pour désigner un canidé), des dénominations lexicales en emploi non standard, notamment en emploi métaphorique ${ }^{14}$ (Paul est vraiment un chien), ou même des formes référentielles ad hoc, comme chose à emporter de la maison en feu dans l'exemple du PIANO évoqué plus haut (Barsalou 1983, Dubois \& Mondada 1995).

On remarque d'emblée que, bien que cette approche et la précédente recourent aux mêmes termes de catégories et de catégorisation, elles ne traitent pas des mêmes objets linguistiques. La sémantique lexicale et cognitive ${ }^{15}$ s'intéresse aux catégories en tant qu'elles sont enregistrées par du lexique et dans des emplois strictement lexicaux, là où les processus de catégorisation étudiés par la seconde approche ne relèvent pas exclusivement du lexique. Or, la nature lexicale ou non lexicale des formes portant la catégorisation n'est pas sans conséquence lorsque l'on analyse cette opération d'un point de vue langagier, telle qu'elle est mise en œuvre dans les discours.

Bien que le dernier exemple cité concernant le piano soit exploité hors contexte par les auteurs ${ }^{16}$, les approches constructivistes de la référence et les approches ethnométhodologiques de la catégorisation travaillent le plus souvent sur des séquences textuelles ou interactionnelles dans lesquelles diverses formes de catégorisation successives apparaissent. Les auteurs défendent l'idée que toutes ces catégorisations concourent au même titre à la construction de l'objet de discours. Dès lors, ces travaux s'intéressent tout autant à la catégorisation initiale d'un objet de discours qu'aux diverses recatégorisations qu'il subit dans le fil du discours (Apothéloz \& Reichler-Béguelin 1995, Dubois \& Mondada 1995, Berthoud 1999). Nous montrerons pour notre part que toutes les formes instaurant des catégorisations ne jouent pas le même rôle dans la construction textuelle de la référence, et qu'il est essentiel de mettre en lumière leurs contraintes d'emploi pour rendre compte des fonctionnements de la catégorisation dans les textes. 
C'est dans l'optique constructiviste évoquée qu'Apothéloz \& Reichler-Béguelin (1995) exploitent ce poème de Ponge :

L'huître, de la grosseur d'un galet moyen, est d'une apparence plus rugueuse, d'une couleur moins unie, brillamment blanchâtre. C'est un monde opiniâtrement clos. Pourtant, on peut l'ouvrir : il faut alors la tenir au creux d'un torchon, se servir d'un couteau ébréché et peu franc, s'y reprendre à plusieurs fois. (F. Ponge, "L’huître », Le parti pris des choses)

Dans leurs analyses, les auteurs montrent que l'objet de discours est construit tout autant par la forme l'huître que par le SN un monde. Les deux syntagmes joueraient un rôle équivalent dans la construction de la référence à l'objet de discours. Or, il ne nous semble pas que ce soit le cas. L'approche constructiviste se fonde sur une conception du discours que l'on peut qualifier de cognitive : le discours est conçu dans cette perspective comme un espace de construction de représentations. Mais pour analyser la configuration des discours, il faut selon nous prendre en compte les propriétés linguistiques, tant sémantiques que formelles, des expressions référentielles qui y sont convoquées, et non pas seulement leur valeur cognitive. Ainsi, nous pensons que dans ce passage, les deux SN ne contribuent pas de la même manière à la construction de la référence. Plus précisément, ces rôles différenciés tiennent pour nous aux propriétés sémantico-discursives des formes, aux enchaînements textuels, à la structuration informationnelle de la séquence, ainsi qu'aux emplois syntaxiques des formes.

Du point de vue sémantico-discursif, les deux formes nominales n'apparaissent pas dans les mêmes emplois, et ne fournissent pas le même accès au référent. Huître est ici en emploi classifiant : il permet d'identifier le type de référent visé, la catégorisation qu'opère cette forme étant fondée sur les propriétés extralinguistiques du référent. En empruntant une expression exploitée dans le cadre de la théorie des référents évolutifs (Achard-Bayle 2001 a) - mais sans nous situer dans cette perspective -, nous pouvons dire que la catégorisation se fonde sur l'identité sortale du référent, à savoir son appartenance à telle espèce naturelle ${ }^{17}$. L'emploi du nom huître en début de texte inscrit donc l'objet visé dans une taxinomie de savoirs sur le monde. La dénomination apparaît ici en emploi strictement lexical, c'est-à-dire qu'elle est utilisée dans son champ d'applicabilité référentielle enregistré dans le système de la langue.

La forme monde est au contraire en emploi qualifiant: elle donne à voir l'objet de discours depuis un point de vue spécifique. La valeur classifiante du nom monde est désactivée au profit d'une valeur qualifiante : il s'agit ici pour le poète non pas d'identifier la chose dont il parle, mais d'en bâtir une certaine représentation, de la donner à voir sous un jour particulier.

Remarquons d'ailleurs que cette représentation se veut originale, subjective, poétique, etc., ce qui n'est pas indifférent. La dimension éventuelle de littérarité est à prendre en compte dans l'analyse des modes de construction de la référence mis en œuvre dans un texte. Un discours littéraire ne construit pas les objets de discours de la même manière qu'un texte non littéraire. Ce n'est donc pas un hasard si nous trouvons dans ce type de passages des noms en emploi qualifiant, avec désactivation de la valeur classifiante : pour le poète, il ne s'agit certainement pas de fournir une description physique de l'huître, mais de la donner à voir sous un jour inédit. De fait, il importe de tenir compte de l'inscription d'un texte dans le champ littéraire pour analyser les processus référentiels.

Or, les valeurs d'emploi classifiantes et qualifiantes sont liées à la structure informationnelle des énoncés ${ }^{18}$ et aux structures syntaxiques dans lesquelles apparaissent les formes nominales. L'inscription de la forme nominale dans les énoncés, en position thématique ou attributive, influe sur sons fonctionnement sémantico-référentiel, et active une valeur classifiante ou qualifiante. Dans cette description, les deux SN n'apparaissent pas dans les mêmes positions au sein des énoncés, ce qui n'est pas indifférent du point de vue référentiel. L'huître apparaît en position thématique (il désigne la chose que pose l'énonciateur afin d'en parler) et strictement référentielle (il opère bien la sortie vers l'extralinguistique en désignant l'objet du monde lui-même), là où un monde apparaît dans une structure attributive (c'est un $x$ ) : il appartient au prédicat, ne désigne pas directement l'objet, mais lui attribue des propriétés. Son rôle n'est donc pas de classifier l'objet de discours, mais de le caractériser. 
Dès lors, il faut relier ces faits à un dernier niveau, celui de la séquentialité. En effet, les deux formes de nomination ne sont pas interchangeables dans l'ordre du texte. La séquence suivante serait mal construite, et plus difficilement interprétable :

? Le monde, de la grosseur d'un galet moyen, est d'une apparence plus rugueuse, d'une couleur moins unie, brillamment blanchâtre. C'est une huître opiniâtrement close.

Le monde ne peut apparaître en position thématique en début de séquence, à moins de désigner un autre référent (non plus un coquillage, mais un monde, hors d'un emploi métaphorique) : il n'est pas apte à identifier l'objet dont parle l'énonciateur, précisément parce que dans cette séquence il ne peut assumer une valeur classifiante ${ }^{19}$. Sa valeur de classification se trouve au contraire désactivée au profit d'une valeur strictement qualifiante, du fait de son emploi métaphorique. Le recours à cette forme en début de séquence textuelle ne permettrait pas de garantir l'intercompréhension.

Le lieu d'apparition de la forme classifiante dans la séquence n'est pas non plus anodin. La place d'une forme au sein de la séquence textuelle, par rapport aux informations déjà construites précédemment, influe selon nous sur son fonctionnement sémantico-référentiel. Au sein de la séquence descriptive, huître joue le rôle de thème-titre (Adam \& Revaz 1989, Adam 2005 a), c'est-à-dire de pivot nominal autour duquel la séquence descriptive s'organise. Il reprend d'ailleurs le titre du poème lui-même. Posant l'objet que l'énonciateur va décrire dans la suite (il est donc logique qu'il apparaisse en position thématique dans la suite d'énoncés), il confère à la séquence sa cohésion sémantico-référentielle. Il correspond donc à un point de référence stable, à partir duquel vont pouvoir être prédiquées des propriétés multiples, qui peuvent notamment apparaître sous la forme d'expressions nominales qualifiantes.

Il importe donc d'analyser les formes au sein des enchaînements textuels. Sur ce plan, Apothéloz \& Reichler-Béguelin (1995) commentent l'anaphore pronominale la (il faut alors la tenir au creux d'un torchon), apparemment atypique puisqu'elle apparait après un monde, et indiquent que cette anaphore " ignore la recatégorisation de l'huître comme monde » (253). Or, cette reprise n'a rien d'étonnant : elle a bien pour antécédent le SN initial l'huître, et non le second un monde, ce qui tient tant à la dimension syntaxique et informationnelle (au fait que un monde soit en position prédicative) qu'à la dimension sémantico-référentielle (c'est bien l'huître qui indexe le référent et l'identifie) ${ }^{20}$.

Ces faits montrent que toutes les formes nominales ne jouent pas le même rôle dans la construction de la référence, et qu'il est nécessaire de prendre en compte la dimension proprement séquentielle de cette dernière.

Nous avons pu vérifier l'hypothèse selon laquelle l'ordre d'apparition des expressions référentielles est contraint au sein des séquences textuelles, à partir d'un corpus de séquences descriptives extraites de récits de voyage (Guérin 2011). Nous y avons observé que ces séquences répondent à une configuration typique. Construites de manière à ce que le lecteur, supposé ne pas avoir de connaissance directe des réalités étrangères décrites dans le texte, puisse les identifier, elles remplissent généralement les deux conditions suivantes : (1) toute séquence descriptive est construite autour d'un thème-titre, qui apparaît à son initiale ; (2) le thème-titre est une forme en emploi classifiant assurant l'identification de l'objet.

L'extrait suivant est représentatif de cette configuration :

Le costume des Maures consiste, pour les riches, en un drâh, tunique de guinée qui leur descend aux jarrets, et dont les manches, aussi larges que le corps, tombent jusqu'à terre. Une culotte faite de dix coudées de guinée les couvre depuis la ceinture jusqu'aux genoux; une pagne complète le vêtement; ils la mettent par-dessus la tunique, et quelquefois sur la tête, en turban ; ils portent rarement des sandales. Ceux qui n'ont pas les moyens d'acheter une tunique portent simplement un coussabe ${ }^{\mathrm{a}}$ fait de cinq coudes de guinée.

a. On nomme coussabe une pièce d'étoffe de deux aunes de long sur trois quarts au moins de large, pliée en deux, et les laizes cousues ensemble, en laissant par le haut des ouvertures pour passer les bras ; on en fait une autre au milieu de l'étoffe pour 


\section{passer la tête. C'est une chemise sans col et sans manches. (R. Caillié, 1830, Voyage à Tombouctou)}

La séquence s'ouvre d'emblée sur l'attribution d'un thème-titre, le costume des Maures, qui pose l'objet décrit ; cette configuration correspond à ce qu'Adam \& Revaz (1989) décrivent sous le terme d'ancrage. Une fois posé ce thème-titre fonctionnant comme holonyme (Cruse 1986), les différentes pièces constituant l'habillement sont détaillées par des méronymes, qui font à leur tour l'objet de prédications, détaillant les propriétés de ces pièces de vêtements au fur et à mesure de la séquence.

Le thème-titre introduit en début de séquence est classifiant en ce qu'il donne des informations sur l'identité du référent. Il permet ici d'indiquer dans quel type de hiérarchie catégorielle le référent s'inscrit (celle des pièces d'habillement): l'identification globale est assurée, et spécifiée par le complément déterminatif des Maures. Le thème-titre assure dès lors l'intercompréhension et confère à la séquence descriptive son interprétabilité. Notons que dans le corpus exploité, on ne relève aucune séquence descriptive qui ne comporterait pas de terme classifiant; indexer l'identité d'un référent, indiquer dans quel type de hiérarchie catégorielle il s'inscrit semble être une condition nécessaire pour garantir l'intercompréhension et pour que les séquences descriptives soient interprétables.

Ainsi, toutes les catégorisations ne sont pas équivalentes au sein de ce type de séquences textuelles. Les formes en emploi classifiant jouent un rôle prééminent dans la construction de la référence, en ce qu'elles permettent au co-énonciateur d'identifier de quoi le texte parle. Elles jouent un rôle essentiel dans la construction d'une représentation discursive et dans l'interprétabilité du texte. Les formes non classifiantes ne concourent à la catégorisation de l'objet de discours que sur un second plan.

Pour le montrer, nous allons compléter l'analyse des emplois classifiants et non classifiants par celui des modes de saturation des formes référentielles.

\section{Modes de saturation référentielle des formes nominales}

En effet, toutes les expressions nominales au sein d'un texte n'impliquent pas les mêmes procédures pour se voir assigner un référent, certaines étant saturées de manière autonome, d'autres ne pouvant l'être que de manière relative, ce qui confirme l'idée de modes diversifiés de construction de la référence.

Pour illustrer ce propos, nous reviendrons sur l'exemple du savon de Ponge évoqué plus haut :

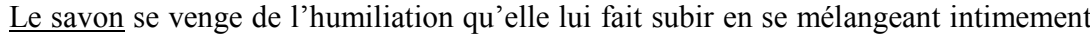
à l'eau, en s'y mariant de la façon la plus ostensible. Cet oeuf, cette plate limande, cette petite amande se développe rapidement en poisson chinois. (F. Ponge, « Le savon », Le parti pris des choses)

Le problème que nous posons ici se rapproche dans une certaine mesure de la différence, établie au sein des études portant sur les expressions référentielles telles que les SN désignant des objets ou des personnes, ou encore les expressions temporelles et spatiales (par exemple Kerbrat-Orecchioni 2002), entre référence absolue et référence relative. Dans cette perspective, la question concerne notamment la sélection du référent singulier d'un $\mathrm{SN}$ : on se demande si sa référence est saturée de manière autonome, relativement au contexte situationnel, ou encore par appui sur le cotexte. La question est donc notamment liée à des problèmes de détermination. Tout en nous interrogeant sur des problèmes de référence, nous déplaçons ici l'objet par rapport à cette perspective: nous nous demandons si l'identification de la catégorie (et non plus celle du référent singulier) s'opère de manière autonome ou non, c'est-à-dire sans recours à une procédure complémentaire d'appui sur le cotexte ${ }^{21}$.

Pour analyser la catégorisation dans l'extrait cité, il n'est pas souhaitable de mettre sur le même plan toutes les formes nominales savon, euf, limande, amande. Cela tient aux différents types de phénomènes que nous avons mis en lumière pour l'extrait précédent, aux valeurs sémantiques d'emploi des formes (savon est en emploi classifiant, alors que les autres noms sont ici métaphoriques et donc en emploi qualifiant), mais aussi aux différents degrés d'autonomie référentielle des expressions nominales. Une forme nominale comme savon se suffit à elle-même pour l'identification du type référentiel, et donc pour 
l'interprétation de l'énoncé par le co-énonciateur. De manière plus générale, les formes classifiantes se suffisent à elles-mêmes, en ce qu'elles instaurent la référence de manière autonome : elles ne nécessitent pas la présence d'une autre forme de nomination du même référent dans le cotexte pour que le lecteur sache de quel type d'objets il est question.

Par contraste, les formes nominales $2 u f$, limande, amande opèrent des « catégorisations » sur la base d'un référent déjà classifié par savon, forme qui assure l'identification. La reprise par une métaphore (non identifiante) ne peut se faire que sur la base d'un référent déjà classifié. À l'inverse, les formes non classifiantes nécessitent un appui sur une autre forme présente dans le cotexte, pour que le lecteur puisse se représenter le type d'objets visé.

Si savon apparaissait seul dans la séquence pour catégoriser le référent, il garantirait l'intercompréhension - il opère donc une catégorisation autonome. Les formes suivantes ne peuvent pas fonctionner de manière autonome vis-à-vis de l'identification du type de référents. Ce fait sémantique converge avec leur emploi textuel au sein de SN anaphoriques de l'antécédent le savon.

Ainsi, selon nous, toutes les catégorisations ne sont pas équivalentes sur le plan référentiel, ni susceptibles d'apparaître dans les mêmes conditions. Nous pensons que les approches qui supposent que toutes les catégorisations s'équivalent pour construire l'objet de discours et qui mettent dès lors l'accent sur l'instabilité foncière des catégories, ont tendance à lisser ces différences de fonctionnement. En outre, l'insistance sur l'instabilité des catégorisations tient, nous semble-t-il, à une prise en compte insuffisante des contraintes liées à la textualité et de l'ordre de la séquentialité dans la construction de la référence.

\section{Un amalgame fréquent entre identification et qualification}

De fait, ce type d'approches opère, pensons-nous, une confusion entre deux opérations différentes, qu'il apparaît essentiel de distinguer dès lors qu'il s'agit de référence à des concrets : l'identification et la qualification. Ces deux types de procédures ne sont pas assimilables. Or, le terme de catégorisation, employé pour renvoyer tantôt à l'une, tantôt à l'autre de ces procédures, entretient la confusion. C'est là une explication des divergences de conception de la catégorisation entre l'approche sémanticoréférentielle d'une part, et les approches constructiviste et ethnométhodologique d'autre part : la première parle surtout de catégorisation identifiante, là où les secondes mettent les catégorisations identifiantes et qualifiantes sur le même plan, et lissent les différences de procédures sous-jacentes.

Une telle conception, qui ne prend en compte ni l'organisation hiérarchique de la séquence, ni son déroulement, a pour conséquence de placer toutes les formes sur le même plan du point de vue de la construction de la référence, là où nous pensons que toutes les formes n'interviennent pas de la même manière ni dans la même mesure dans la construction de l'objet de discours. On gagnerait dès lors à distinguer deux processus différents dont il peut être rendu compte sous le terme de catégorisation:

(1) Dans le premier cas, la forme nominale indique l'identité sortale du référent, c'est-à-dire qu'elle donne des informations sur la classe d'appartenance du référent (dans l'exemple de « L'huître » de Ponge, l'espèce naturelle dont il relève), ce classement s'effectuant sur la base de propriétés définitoires stables et intersubjectivement partagées. De telles formes en emploi classifiant jouent un rôle d'identification ; la catégorisation mise en œuvre permet de savoir de quel type d'objets il est question dans une séquence textuelle.

Du point de vue cognitif et discursif, les formes classifiantes possèdent un statut particulier vis-à-vis des connaissances partagées et de l'intersubjectivité : elles garantissent l'intercompréhension, et permettent au co-énonciateur d'identifier le type d'objet visé. Ceci est d'autant plus nécessaire dans le discours écrit, puisque le lecteur n'a pas d'accès direct à la situation et au référent ${ }^{22}$. Il importe donc qu'une forme remplisse ce rôle d'identification au sein de la séquence ${ }^{23}$.

Pour l'exemple de la description de l'huître par Ponge, l'emploi de la dénomination huître assure cette identification. 
(2) Le deuxième type de catégorisation, marqué par une forme non classifiante (monde dans l'exemple en question), ne donne pas d'informations sur l'identité du référent, et ne constitue pas une dénomination intersubjectivement partagée du type visé. Il s'agit d'une catégorisation ad hoc, dont le rôle discursif n'est pas d'identifier l'objet, mais de le qualifier; la forme permet de bâtir une représentation spécifique du référent, en le présentant depuis un point de vue particulier. Le type de catégorisation concerné ne met pas l'accent sur la place du référent dans l'organisation du réel, mais sur des propriétés accessoires que l'observateur sélectionne, au sein d'une représentation subjective.

Cette distinction débouche sur une autre remarque, qui touche à l'autonomie des formes de référence. Si les formes du premier type peuvent apparaître seules dans une séquence textuelle et suffisent à identifier le référent - la catégorisation fonctionne alors de manière autonome -, ce n'est pas le cas des secondes. Pour identifier le type de référents désigné par les formes de la deuxième série, il est nécessaire de s'appuyer sur une autre forme de catégorisation présente dans le cotexte; la catégorisation s'opère donc de manière relative. De fait, ces formes n'apparaissent pas seules : elles s'appuient sur une autre forme de catégorisation - identifiante celle-là -, et, comme c'est le cas dans l'exemple du savon, elles apparaissent souvent en position de reprise, et non pour la catégorisation initiale du référent.

Cet extrait du carnet de route où Gide évoque un fruit exotique le montre bien :

Du sable, presque uniquement agrémenté par cette étrange plante gris-vert dont enfin je puis voir le fruit : un beignet énorme, bivalve, tenant suspendu en son centre, au milieu d'une matière feutrée, filigranée, un paquet de graines. (A. Gide, 1927, Voyage au Congo)

La désignation métaphorique un beignet ne pourrait pas apparaître comme forme initiale de catégorisation pour ce fruit (*cette étrange plante gris-vert dont enfin je puis voir le beignet). Elle ne peut être employée que dans un second temps, une fois que l'identification de l'objet au moyen d'une forme classifiante a été produite. De fait, la désignation métaphorique n'est pas identifiante; elle assure une fonction de visualisation, en mettant l'accent non sur l'appartenance catégorielle du référent, mais sur sa morphologie, qu'elle représente de manière analogique. Elle suppose la présence d'une autre forme qui la précède et assure l'identification du référent.

Ainsi, à partir du corpus de récits de voyage que nous avons étudié, nous avons pu observer qu'au sein des séquences descriptives, les formes référentielles se répartissent très majoritairement de la manière suivante :

(1) une première catégorisation par une forme classifiante, assurant la fonction d'identification ;

(2) une « catégorisation » par une forme qualifiante, attribuant des propriétés supplémentaires, sur la base d'un référent déjà classifié.

Pour être généralisée à d'autres types de séquences textuelles, une telle hypothèse demanderait à être vérifiée sur des corpus diversifiés; sans pouvoir à ce stade de l'analyse parler de contrainte textuelle générale, on peut envisager qu'il s'agisse d'une tendance massive dans la construction textuelle de la référence.

Nous ferons une dernière remarque sur les analyses constructivistes et sur les modèles de la catégorisation nominale. Les exemples d'analyses que nous avons cités plus haut s'appuient sur des catégorisations de référents concrets. Or, la conception de l'objet de discours sous-jacente aux approches constructivistes (il serait purement constitué de représentations mentales) semble convenir davantage aux objets sociaux ${ }^{24}$ (Searle 1995) qu'aux référents concrets, qui ne sont pas de purs êtres de discours. Leur identité extralinguistique intervient partiellement dans les procédures de catégorisation. Par contraste, la délimitation entre identification et qualification est moins marquée pour les objets sociaux, et il y a recouvrement partiel entre les deux opérations. Cette remarque rejoint celles faites, dans une perspective différente, par Lärsson $(1997,2008)$ et Kleiber (1997) au sujet de la construction de l'intersubjectivité. Selon ces auteurs, il existe des différences de stabilité sémantique selon les domaines d'entités : les objets concrets ont a priori une stabilité intersubjective plus forte que les objets sociaux; ainsi, l'idée d'une renégociation perpétuelle du sens serait valable surtout pour les domaines présentant un degré de stabilité 
peu élevé (Lärsson prend pour exemple un lexème comme amour, dont le sens est éminemment soumis à variation).

De fait, une part de la difficulté quant à l'application des thèses constructivistes pour analyser la référence telle qu'elle est mise en œuvre dans les discours nous semble résider dans le fait de recourir, pour décrire les procédures de catégorisation des concrets, opération dans laquelle l'extralinguistique (et, ne leur en déplaise, l'« ontologie » du référent) intervient de manière importante, à des modèles qui semblent davantage adaptés pour rendre compte de la catégorisation des objets sociaux, où la part des représentations est majoritaire. Dans le cas de la catégorisation des concrets, et tout particulièrement au sein d'un texte écrit, la procédure d'identification est centrale, et effectuée par les formes classifiantes.

\section{Conclusion : Vers un modèle modulaire de la catégorisation}

L'approche que nous avons tenté de développer dans cet article se caractérise ainsi comme un modèle modulaire de la catégorisation nominale, articulant quatre niveaux de contraintes, qui relèvent :

\section{(1) de l'extralinguistique :}

Il importe selon nous de tenir compte de l'extralinguistique dans l'analyse des faits de catégorisation, tout particulièrement lorsqu'il s'agit de référence aux concrets. La part des représentations dans les phénomènes de catégorisation - privilégiée dans la plupart des approches discursives de la catégorisation -, si elle est bien attestée, nous semble cependant circonscrite. Contrairement aux approches qui défendent l'idée que toutes les catégorisations se valent, en quelque sorte, qu'un objet de discours peut être catégorisé de manière libre, et que les catégorisations prototypiques n'ont pas de statut particulier, nous pensons que l'identité du référent influe bien sur les pratiques de catégorisation. Les catégorisations indexées sur l'identité sortale possèdent un statut particulier vis-à-vis des connaissances partagées et de l'intersubjectivité : ce sont elles qui assurent l'intercompréhension. Ainsi, dans la catégorisation, tout n'est pas affaire de représentations; l'extralinguistique intervient bien dans ces processus.

(2) du fonctionnement linguistique des formes nominales en contexte :

Tout en travaillant sur la catégorisation telle qu'elle est mise en œuvre en discours, nous pensons que l'ordre de la langue et des fonctionnements systémiques des formes reste prégnant et a une influence sur la construction de la référence. Ainsi, nous avons tenu compte des fonctionnements sémantico-référentiels différenciés des formes nominales (en emploi classifiant vs qualifiant), de certains de leurs emplois syntaxiques, de la structure informationnelle des énoncés. Toutes ces contraintes font apparaître des types de catégorisation différenciés. Il nous semble qu'un certain nombre des malentendus théoriques autour de la notion de catégorisation (notamment entre la sémantique lexicale et les approches discursives de la catégorisation) tient au fait que les deux ensembles d'approches ne traitent pas des mêmes phénomènes sous le même terme ${ }^{25}$.

(3) de la séquentialité :

Pour rendre compte de la construction de la référence et de la manière dont y interviennent les formes instaurant des catégorisations, il importe d'observer la textualité des séquences, en particulier descriptives, dans le détail de son organisation. Nous avons notamment insisté sur le fait que les différents types d'expressions référentielles ne sont pas susceptibles d'apparaître aux mêmes places au sein de la séquence, en raison de procédures sémantico-référentielles sous-jacentes spécialisées. Il convient donc d'observer l'ordre de la séquentialité, les reprises, les enchaînements (qui valident ou non les catégorisations initiales), et les contraintes qui pèsent ainsi sur la construction textuelle de la référence. 
(4) de l'ordre du discours :

La constitution des discours est également informée par des contraintes, au premier rang desquelles la nécessaire construction de l'intercompréhension. Les modèles insistant sur l'instabilité foncièrement des représentations sémantiques tiennent insuffisamment compte du fait que les discours "fonctionnent» malgré tout, qu'ils arrivent à construire des objets globalement stables. C'est qu'ils se fondent largement sur le partagé, celui-ci tenant d'une part au fait que nous partageons une grande partie de notre expérience sur le monde extralinguistique, et d'autre part au fait que nous disposons de formes linguistiques dont le sens est grosso modo suffisamment stable pour que nous puissions renvoyer à ces référents sans avoir à tout renégocier systématiquement.

À ce type de contraintes, il faudrait ajouter celles qui tiennent aux formatages impliqués par les types et genres de discours dans lesquels se coule l'opération de référence. Nous n'avons pu faire, dans le cadre de cet article, que des remarques ponctuelles sur ce point (on a pu remarquer par exemple que la référence dans les récits de voyage est davantage configurée par la didacticité, là où les extraits littéraires font plus de place à la subjectivité dans la catégorisation), mais il serait intéressant de vérifier une telle hypothèse à partir de corpus diversifiés.

Ainsi, tout en ayant des objets similaires à ceux des approches constructivistes, nous aboutissons à des conclusions assez différentes sur les procédures sous-jacentes à la construction textuelle de la référence et à l'usage des formes nominales pour la catégorisation. Plutôt que pour une approche cognitive du discours centrée sur la construction de représentations subjectives et mouvantes, nous optons pour une conception du discours qui met l'accent sur les contraintes linguistiques et extralinguistiques et sur les facteurs de stabilisation de la référence.

\section{Références bibliographiques}

ACHARD-BAYLE, G. (2001 a), Grammaire des métamorphoses: référence, identité, changement, fiction. Bruxelles : Duculot.

ACHARD-BAYLE, G. (2001 b), «Faits de langue, de texte... Effets de fiction : des désignations dans les 'dispositifs fictionnels'». in L'effet de fiction, colloque en ligne Fabula [en ligne], $<$ http://www.fabula.org/effet/interventions/1.php>. [Page consultée le 13/01/2011]

ADAM, J.-M. (2005 a), Les textes : types et prototypes. Récit, description, argumentation, explication et dialogue. Paris : Nathan Université.

ADAM, J.-M. (2005 b), La linguistique textuelle. Introduction à l'analyse textuelle des discours. Paris : Armand Colin.

ADAM, J.-M., REVAZ, F. (1989), « Aspects de la structuration du texte descriptif : les marqueurs d'énumération et de reformulation ». Langue française, vol. $81, \mathrm{n}^{\circ} 1$, pp. 59-98.

APOTHÉLOZ, D., REICHLER-BÉGUELIN, M.-J. (1995), «Construction de la référence et stratégies de désignation ». TRANEL, ${ }^{\circ} 23$, pp. 227-271

BARSALOU, L. (1983), «Ad-hoc categories », Memory and Cognition. n¹1, pp. 211-227.

BERGER, P. L., LUCKMANN, T. (1966), The Social Construction of Reality. New York : Doubleday.

BERTHOUD, A.-C. (1999), "Recatégorisation des objets au fil du discours ». in A. Deschamps, J. GuilleminFlescher éd., Les opérations de détermination : qualification / quantification. Paris : Ophrys, pp. 185-198.

BRANCA-ROSOFF, S. (2007), « Approche discursive de la nomination/dénomination ». in G. Cislaru et al. éd., pp. $13-22$.

CADIOT, P., HABERT, B. (1997), « Aux sources de la polysémie lexicale ». Langue française, n¹13, pp. 3-11.

CADIOT, P., LEBAS, F. (2003), « La constitution extrinsèque du référent ». Langages, $\mathrm{n}^{\circ} 150$, pp. 3-8.

CADIOT, P., NÉMO, F. (1997), «Propriétés extrinsèques en sémantique lexicale ». French language studies, $\mathrm{n}^{\circ} 7$, pp. 127-146.

CALABRESE, L. (2007), « Quel(s) objet(s) de discours se dissimule(nt) sous la dénomination voile ? ». in G. Cislaru et al. éd., pp. 135-148.

CHAROLLES, M., SCHNEDECKER, C. (1993), «Coréférence et identité. Le problème des référents évolutifs ». Langages, $\mathrm{n}^{\circ} 112$, pp. 106-126.

CISLARU, G., GUÉRIN, O., MORIM, K., NEE, É., PAGNIER, T., VENIARD, M. éd. (2007), L'acte de nommer. Une dynamique entre langue et discours. Paris : Presses Universitaires de la Sorbonne nouvelle. 
CONSTANTIN DE CHANAY, H. (2001), «La dénomination : perspective discursive et interactive ». Cahiers de praxématique, $\mathrm{n}^{\circ} 36$, pp. 169-188.

CRUSE, D. A. (1986), Lexical semantics. Cambridge : Cambridge University Press.

DELEPAUT, G., DUBOIS, D., MZALI, M., GUERRAND, S. (2007), «Dénominations et représentations sémantiques du trajet de train ». in G. Cislaru et al. éd., pp. 53-66.

DE MULDER, W., SCHNEDECKER, C. éd. (2001), Les référents évolutifs. Entre linguistique et philosophie. Paris : Klincksieck.

DUBOIS, D. éd. (2010), Le sentir et le dire : concepts et méthodes en psychologie et linguistique cognitives. Paris : L'Harmattan.

DUBOIS, D., MONDADA, L. (1995), «Construction des objets de discours et catégorisation : une approche des processus de référenciation ». TRANEL, $\mathrm{n}^{\circ} 23$, pp. 273-302.

DUBOIS, D., POITOU, J. (1999), «Catégories sémantiques et cognitives : une étude expérimentale en sémantique lexicale ». Cahiers de lexicologie, $\mathrm{n}^{\circ} 74$, pp. 15-27.

GARFINKEL, H. (1967), Studies in Ethnomethodology. Englewood Cliffs, Prentice Hall.

GUÉRIN, O. (2011), Nomination et catégorisation des realia exotiques dans les récits de voyage (Afrique noire, de la fin du $18^{e}$ siècle à 1960) : une approche sémantico-discursive. Thèse, Paris : Université Paris 3.

GUÉRIN, O. (À paraître), «Dénomination et catégorisation linguistique : entre ontologie et représentations ». in G. Petit éd., La dénomination. Paris : Honoré Champion, 20 p.

KERBRAT-ORECCHIONI, C. (2002), L'énonciation. De la subjectivité dans le langage. Paris : Armand Colin.

KLEIBER, G. (1990), La sémantique du prototype. Catégorisation et sens lexical. Paris : Presses Universitaires de France.

KLEIBER, G. (1991), « Hiérarchie lexicale : catégorisation verticale et termes de base ». Sémiotiques, n ${ }^{\circ}$, pp. 35-57.

KLEIBER, G. (1997), «Sens, référence et existence : que faire de l'extra-linguistique ? ». Langages, ${ }^{\circ} 127$, pp. 9-37.

KLEIBER, G. (2001 a), «Remarques sur la dénomination ». Cahiers de praxématique, n³6, pp. 21-41.

KLEIBER, G. (2001 b), L'anaphore associative. Paris : PUF.

KLEIBER, G., SCHNEDECKER, C., TYVAERT, J.-E. éd. (1997), La continuité référentielle. Paris : Klincksieck.

LÄRSSON, B. (1997), Le bon sens commun: remarques sur le rôle de la (re)cognition intersubjective dans l'épistémologie et l'ontologie du sens. Lund : Lund University Press.

LÄRSSON, B. (2008), «Le sens commun ou la sémantique comme science de l'intersubjectivité humaine », Langages, $\mathrm{n}^{\circ} 170$, pp. $28-40$.

MILNER, J.-C. (1978), De la syntaxe à l'interprétation. Quantités, insultes, exclamations. Paris : Le Seuil.

MONDADA, L. (1994), Verbalisation de l'espace et fabrication du savoir. Approche linguistique de la construction des objets de discours. Lausanne, Université de Lausanne.

MONDADA, L. (1995), «La construction discursive des objets de savoir dans l'écriture de la science », Réseaux, $\mathrm{n}^{\circ} 71$, pp. $55-77$.

MONDADA, L. (1997), «Processus de catégorisation et construction discursive des catégories », in D. Dubois éd., Catégorisation et cognition : de la perception au discours. Paris : Kimé, pp. 291-313.

MORTUREUX, M.-F. (1993), « Paradigmes désignationnels ». Semen, nº, pp. 121-142.

NEE, É. (2009), Sûreté, sécurité, insécurité. D’une description lexicologique à une étude du discours de presse : la campagne électorale 2001-2002 dans le quotidien Le Monde. Thèse, Paris : Université Paris 3.

NYCKEES, V. (1997), «Catégories sémantiques et historicité des significations ». Histoire, Épistémologie, Langage, $n^{\circ} 19 / 1$, pp. 97-119.

NYCKEES, V. (1998), La sémantique. Paris : Belin.

PETIT, G. (1995), « La désignation de 'timbre-poste' ». Carnets du CEDISCOR, n³, pp. 27-42.

PETIT, G. (2009), La dénomination : approches lexicologique et terminologique. Louvain-Paris : Peeters.

REBOUL, S. (1993), «La terminologie télématique : problèmes de reformulations discursives autour du concept de vidéographie ». In A. Ali Bouacha et al. éd., Parcours linguistiques de discours spécialisés, Berne, Peter Lang, pp. 23-32.

ROSCH, E. (1973), « Natural categories », Cognitive Psychology. Vol. 4, n³, pp. 328-350.

ROSCH, E. (1976), «Classification d'objets du monde réel : origines et représentations dans la cognition ». In $\mathrm{S}$. Ehrlich, E. Tulving éd., Bulletin de psychologie, numéro spécial, Paris : [s.n.], pp. 242-250.

ROSCH, E. (1978), « Principles of categorization ». In E. Rosch, B. Lloyd éd. (1978), Cognition and Categorization, Hillsdale, Lawrence Erlbaum Associates, pp. 27-48.

SACKS, H. (1992), Lectures on Conversation. Oxford, Basil Blackwell.

SCHNEDECKER, C. (1998), Nom propre et chaînes de référence. Metz, Université de Metz ; Paris : diff. Klincksieck.

SEARLE, J. R. (1995/1998), La construction de la réalité sociale. Paris : Gallimard.

SIBLOT, P. (2001), « De la dénomination à la nomination. Les dynamiques de la signifiance nominale et le propre du nom ». Cahiers de praxématique, n³6, pp. 189-214. 
SITRI, F. (1998), Un modèle d'objet de discours dialogique, entre thématisation et reprise. Étude de situations trilogales orales. Thèse, Paris : Université Paris 3.

VENIARD, M. (2007), La nomination d'un événement dans la presse quotidienne nationale. Une étude sémantique et discursive : la guerre en Afghanistan et le conflit des intermittents dans le Monde et le Figaro. Thèse, Paris : Université Paris 3.

\footnotetext{
${ }^{1}$ La sémantique référentielle, lexicale et cognitive (initialement Rosch 1973, 1976, 1978, puis notamment Kleiber 1990, 1991, 2001, ou encore Nyckees 1997, 1998) s'intéresse à la catégorisation en tant qu'elle est enregistrée dans les formes lexicales, qui sémiotisent l'organisation des savoirs sur le monde. Dans cette perspective, on s'intéresse principalement aux catégories d'espèces naturelles et d'objets en tant qu'elles sont enregistrées dans le lexique d'une langue.

${ }^{2}$ Voir Kleiber (1997) pour un rappel des débats ayant opposé la théorie des référents évolutifs et les approches constructivistes de la référence.

${ }^{3}$ Les deux approches diffèrent en ce que la sémantique indexicale travaille sur l'unité mot, là où les secondes prennent pour unité d'étude non le mot mais le discours. Tout se centrant sur des mots, la sémantique indexicale dénie l'existence d'une stabilité sémiotique et référentielle, la signification d'un mot ne pouvant s'établir qu'au niveau de l'énoncé, et non en langue.
}

${ }^{4}$ Voir notamment Berger \& Luckmann (1966) pour les fondements de cette approche. Dans la mesure où le constructivisme est un courant représenté par des approches diverses, nous ne prétendons pas rendre compte de l'ensemble de ce paradigme. Cependant, nous nous intéresserons à des études qui, au sein de ce paradigme général, s'intéressent à des problèmes de catégorisation au moyen de formes nominales (notamment Apothéloz \& ReichlerBéguelin 1995, Berthoud 1999), dans la perspective d'une remise en question de la notion de référence. Nous nous situerons par rapport à elles pour illustrer notre propre conception des opérations référentielles.

${ }^{5}$ Voir note 1

${ }^{6}$ Sitri (1998) propose de l'objet de discours une conception moins strictement cognitive, prenant davantage en compte sa dimension syntaxique (il est repérable par des formes de thématisation et de reprise) et dialogique (il est traversé par des discours tenus antérieurement).

${ }^{7}$ Pour Dubois \& Mondada (1995), le terme de référenciation serait préférable à celui de référence dans la mesure où il met l'accent sur le versant procédural de cette opération.

${ }^{8}$ Or, il existe une voie médiane en sémantique, développée notamment par Lärsson (1997, 2008) et Kleiber (1997), celle d'une sémantique ni entièrement objectiviste, ni entièrement subjectiviste. Pour Lärsson (2008), le sens est une propriété intersubjective, c'est-à-dire qu'il ne peut être reconnu que s'il est partagé par au moins deux locuteurs. Cette conception permet de contourner les difficultés de la sémantique objectiviste qui ne peut pas rendre compte des zones de sens instables, mais remet également en cause le postulat constructiviste selon lequel le sens n'est jamais préétabli, et qu'il peut constamment être renégocié en discours.

${ }^{9}$ Dans la lignée des travaux de Garfinkel (1967) et de Sacks (1992).

${ }^{10}$ Notamment Mondada (1994, 1995, 1997), Dubois \& Mondada (1995), Constantin de Chanay (2001).

${ }^{11}$ C'est le cas dans les études que nous évoquons.

12 Tous les exemples que nous empruntons aux études constructivistes, ainsi que les nôtres, renvoient à des descriptions d'objets concrets.

${ }^{13}$ Elle reste cependant présente, puisque la personne visée est aussi rangée dans une classe d'appartenance.

${ }^{14}$ Nous en verrons des exemples dans les extraits analysés dans cette section.

${ }^{15}$ Voir Kleiber (1990) pour une synthèse, et Nyckees $(1997,1998)$.

${ }^{16}$ Ce qui ne permet pas de mettre en lumière les éventuelles contraintes d'emploi pesant sur ces formes, qui, selon nous, ne sont pas susceptibles d'apparaître dans les mêmes contextes situationnels ni dans les mêmes types de cotextes. Ainsi, il n'est pas possible d'analyser le comportement discursif de ces formes, alors même que la perspective dont il est question ici se positionne comme une approche en discours de la catégorisation. 
17 Achard-Bayle (2001 a) exploite pour rendre compte des phénomènes de référence évolutive. Dans cette perspective, l'identité sortale d'un être vivant ou d'un objet concret, c'est son appartenance à telle espèce ou à tel type d'objets, qui constitue une propriété inaliénable d'une entité ; il y a référence évolutive dès lors qu'il y a changement d'identité sortale pour un référent. Ajoutons que cette identité est à concevoir comme indépendante des points de vue particuliers que l'on peut avoir sur l'objet.

${ }^{18}$ Le fait que la place d'une forme au sein des enchaînements textuels à l'intérieur de la séquence et de sa structure informationnelle influe sur son fonctionnement sémantico-référentiel et son aptitude à classifier confirme le fait que classifiance et non classifiance ne sont pas des statuts intrinsèques aux formes, mais sont bien liées aux emplois en discours.

${ }^{19}$ Sa valeur de classification est ici désactivée au profit d'une valeur strictement qualifiante.

${ }^{20}$ Bien que l'on n'ait pas affaire ici à un phénomène d'anaphore entre l'huître et un monde, on peut rapprocher ce phénomène de ce que Schnedecker (1998 : 29) appelle les anaphores passoires :

«Nous qualifierons les anaphores nominales inaptes à conditionner le pronom personnel subséquent d'anaphores passoires. Elles sont l'effet d'une saisie contingente par laquelle le locuteur apporte à un point donné de la chaîne un éclairage nouveau, ponctuel mais non décisif, sur le référent. En sorte que l'interlocuteur n'a pas besoin de garder ce type d'indication en mémoire à long terme et peut l'évacuer aussitôt. »

${ }^{21}$ L'appui sur le contexte situationnel n'est pas pertinent dans notre perspective, puisque nous travaillons sur du discours écrit et différé, où l'interprétation des formes par deixis n'est pas possible.

${ }^{22}$ Par contraste, à l'oral, une forme non autonome du point de vue sémantico-référentiel peut être saturée par appui sur la situation d'énonciation, ce qui n'est pas possible dans le cas du discours écrit.

${ }^{23}$ À moins d'envisager des situations discursives un peu marginales comme l'énigme ou la devinette, où la chose dont on parle n'est pas identifiée explicitement par l'énonciateur, et où c'est le co-énonciateur qui doit, à partir des propriétés indiquées, restituer le type référentiel dont il s'agit, quitte à ce que l'intercompréhension ne soit pas assurée. Mais ce n'est évidemment pas là un type de discours prototypique.

${ }^{24}$ Des objets sociaux tels que guerre (nous empruntons cet exemple à Veniard 2007), insécurité (Nee 2009), affaire $d u$ voile (Calabrese 2007), ville, licenciement / dégraissage (Siblot 2001) ne sont pas indépendants du discours. Leur existence même est problématique, et sujette à débat (Branca-Rosoff 2007). Il est difficile de leur assigner une identité stable, partagée, en dehors des points de vue des sujets et des discours que ces derniers portent sur eux. Ces objets sont au contraire créés par le discours et dépendants des représentations.

${ }^{25}$ Voir Guérin (à paraître) pour une discussion. 